\title{
Safety of Daptomycin in Patients Receiving Hemodialysis
}

\author{
Bruce A. Mueller, Pharm.D., Jason A. Crompton, Pharm.D., Brian J. Donovan, Pharm.D., \\ Sara Yankalev, Ph.D., and Kenneth C. Lamp, Pharm.D.
}

Study Objective. To determine the safety of daptomycin administered using a variety of doses and dosing frequencies in patients receiving intermittent hemodialysis who had probable or confirmed gram-positive infections.

Design. Analysis of data from the Cubicin Outcomes Registry and Experience (CORE), a multicenter, retrospective, observational registry.

Setting. Fifty-four study sites, mostly (46\%) large teaching hospitals.

Patients. Three hundred ninety-three adults in the CORE registry who received intermittent hemodialysis between 2005 and 2008.

Measurements and Main Results. The CORE registry is noninterventional and collects standard-of-care data on daptomycin treatment from health care institutions. Of the 393 patients, 370 (94\%) could be categorized by daptomycin dosing frequency: every 48 hours (251 patients [64\%]), 3 times/week (87 [22\%]), and every 24 hours (32 [8\%]); the remaining 23 (6\%) had unreported dosing frequencies or received a single dose of daptomycin. Three hundred eighty-four patients (98\%) received part of their daptomycin therapy as an inpatient and 129 patients (33\%) received part of their daptomycin therapy in an intensive care setting. The primary infection type was bacteremia (224 patients [57\%]), and the most common pathogen was Staphylococcus aureus (155 patients [39\%]). Thirty-eight adverse events possibly related to daptomycin occurred in 28 patients (7\%); increased blood creatine kinase level (7 patients [1.8\%]) was the most common adverse event. Adverse-event rates were similar across all dosing regimens.

Conclusion. In these patients undergoing hemodialysis, daptomycin was a well-tolerated treatment for gram-positive infections across several doses and dosing frequencies. Further study in prospective trials is warranted.

Key Words: antibiotics, daptomycin, gram-positive infection, hemodialysis, Staphylococcus aureus, vancomycin.

(Pharmacotherapy 2011;31(7):665-672)

Hemodialysis is a life-sustaining treatment for persons with end-stage renal disease. However, the use of hemodialysis is complicated by various issues, including difficulties with vascular access and infection. Infection of any type is the second leading cause of death in patients requiring hemodialysis, with infection causing death in 12$22 \%$ of patients with end-stage renal disease. ${ }^{1}$ Septic syndrome, for example, is common and causes more than $75 \%$ of infection-related deaths in the hemodialysis population. Sepsis-related mortality is $100-300$-fold greater in patients receiving hemodialysis compared with the general population. ${ }^{1,2}$ Most infections in patients receiving hemodialysis are caused by grampositive bacteria, more specifically Staphylococcus aureus, ${ }^{3}$ and the incidence of invasive infection caused by methicillin-resistant S. aureus (MRSA) in the dialysis population is 113-2256-fold greater than that in the general population. ${ }^{4}$

The treatment of infection in patients receiving hemodialysis is complicated by several factors. Since many antibiotics used to treat infections in the hemodialysis population, including infections 
caused by S. aureus, are eliminated primarily through the kidneys, their administration can be challenging in this patient population. In addition, the potential for toxicity may increase as the plasma concentrations of the drug increase due to reduced renal clearance. Vancomycin, which has been the standard therapy for MRSA infection, shares these characteristics. ${ }^{5}$ In addition, vancomycin efficacy appears to be lower against MRSA infections, with elevated vancomycin minimum inhibitory concentration (MIC) values in the susceptible range. ${ }^{6-8}$ Although extremely rare, the first United States reports of vancomycin-resistant $S$. aureus were in patients receiving hemodialysis. ${ }^{9,} 10$ In addition, renal failure is a common independent risk factor for mortality in various infection types and clinical settings. ${ }^{11-13}$ The factors contributing to increased mortality may be antibiotic related or due to patient characteristics that complicate their response to therapy. In either case, additional information on antibiotic use in this population is needed.

Daptomycin (Cubicin; Cubist Pharmaceuticals, Inc., Lexington, MA) is a cyclic lipopeptide that causes rapid, concentration-dependent bacterial cell death against gram-positive organisms. ${ }^{14}$ The activity of daptomycin, vancomycin, and linezolid against $S$. aureus isolates from bacteremic patients receiving hemodialysis is similar, with 99.8- 100\% susceptibility. ${ }^{15}$ Daptomycin has demon-strated similar efficacy to vancomycin and semi-synthetic penicillins in randomized, multicenter trials of complicated skin and skin structure infections and bacteremia and endocarditis, although patients were limited to those with normal to moderately reduced renal function. ${ }^{16,17}$ Pharmacokinetic analysis reveals that daptomycin is more than $90 \%$ bound to plasma proteins and primarily is excreted unchanged by the kidneys in healthy volunteers.

From the College of Pharmacy, University of Michigan, Ann Arbor, Michigan (Dr. Mueller); and Clinical Development and Medical Affairs, Cubist Pharmaceuticals, Inc., Lexington, Massachusetts (Drs. Crompton, Donovan, Yankalev, and Lamp).

Supported by Cubist Pharmaceuticals, Inc.

Presented in part at the annual meeting of the American College of Clinical Pharmacy, Denver, Colorado, October 14-17, 2007.

Manuscript received September 17, 2010. Accepted pending revisions October 25, 2010. Accepted for publication in final form January 23, 2011.

For reprints, visit http://www.atypon-link.com/PPI/loi/phco. For questions or comments, contact Kenneth C. Lamp, Pharm.D., Cubist Pharmaceuticals, Inc., 65 Hayden Avenue, Lexington, MA 02421; e-mail: kenneth.lamp@cubist.com.
In studies of patients receiving hemodialysis, protein binding is similar at $90 \%$, clearance is approximately one third, and the corresponding half-life and area under the concentration-time curve are more than doubled compared with those parameters in patients who have normalto-moderate renal impairment. ${ }^{18-20}$

In patients treated with hemodialysis, the recommended dosing regimen of daptomycin is 4 $\mathrm{mg} / \mathrm{kg}$ every 48 hours for complicated skin and skin structure infections and $6 \mathrm{mg} / \mathrm{kg}$ every 48 hours for $S$. aureus bacteremia or right-sided endocarditis. ${ }^{20}$ When possible, daptomycin should be administered after hemodialysis on hemodialysis days. ${ }^{20}$ This may be difficult when the currently approved every-48-hour dosing regimen falls on days opposite the thrice-weekly hemodialysis schedule commonly used by patients. Consequently, the daptomycin dosing regimen that is used in the real-life management of these patients may fall outside of recommended labeling, since most patients receive intravenous antibiotics during or immediately after their dialysis session.

The objective of this study was to describe the safety profile of daptomycin administered using a variety of doses and dosing schedules in the overall treatment of patients receiving hemodialysis who had probable or confirmed gram-positive infections, by analyzing data from a large, retrospective, multicenter registry.

\section{Methods}

\section{Data Source}

The Cubicin Outcomes Registry and Experience (CORE) is a multicenter, retrospective, noncomparative, observational registry implemented to collect standard-of-care data on daptomycin use in different types of gram-positive infections and patients, including those receiving hemodialysis. ${ }^{21}$ From the time period of 20052008, patient data and clinical outcomes were collected in the United States for the CORE registry. All sites were required to obtain appropriate local or central institutional review board approval, and sites were chosen if they could provide data on a prespecified number of patients and represented a variety of treatment settings, including hospitals and outpatient centers. Patient records were included in the registry if the patients were treated with one or more doses of daptomycin and were not administered daptomycin as part of a clinical trial. ${ }^{21}$ Data were abstracted from patient records 
Table 1. Baseline Demographic and Clinical Characteristics of the Study Patients by Daptomycin Dosing Schedule

\begin{tabular}{|c|c|c|c|c|}
\hline \multirow[b]{2}{*}{ Characteristic } & \multicolumn{3}{|c|}{ Daptomycin Dosing Schedule } & \multirow[b]{2}{*}{$\begin{array}{l}\text { All Patients } \\
\quad(n=393)\end{array}$} \\
\hline & $\begin{array}{l}\text { Every } 48 \text { Hours } \\
(\mathrm{n}=251)\end{array}$ & $\begin{array}{l}3 \text { Times/Week } \\
(\mathrm{n}=87)\end{array}$ & $\begin{array}{l}\text { Every } 24 \text { Hours } \\
(\mathrm{n}=32)\end{array}$ & \\
\hline \multirow[t]{2}{*}{ Weight, median (range), kg } & $74(40-174)$ & $67(36-167)$ & $73(45-122)$ & $73(36-174)$ \\
\hline & \multicolumn{4}{|c|}{ No. (\%) of Patients } \\
\hline \multicolumn{5}{|l|}{$\overline{\operatorname{Sex}}$} \\
\hline Male & $116(46)$ & $33(38)$ & $17(53)$ & $176(45)$ \\
\hline Female & $135(54)$ & $54(62)$ & $15(47)$ & $217(55)$ \\
\hline \multicolumn{5}{|l|}{ Age (yrs) } \\
\hline$\leq 50$ & $72(29)$ & $27(31)$ & $6(19)$ & $112(28)$ \\
\hline $51-65$ & $90(36)$ & $34(39)$ & $17(53)$ & $149(38)$ \\
\hline$\geq 66$ & $89(35)$ & $26(30)$ & $9(28)$ & $132(34)$ \\
\hline \multicolumn{5}{|l|}{$\begin{array}{l}\text { Location } 2 \text { days before } \\
\text { daptomycin administration }\end{array}$} \\
\hline Community & $52(21)$ & $21(24)$ & $12(38)$ & $91(23)$ \\
\hline Hospital & $176(70)$ & $57(66)$ & $18(56)$ & $267(68)$ \\
\hline $\begin{array}{l}\text { Nursing home or extended-care } \\
\text { facility }\end{array}$ & $23(9)$ & $9(10)$ & $2(6)$ & $35(9)$ \\
\hline \multicolumn{5}{|l|}{ Daptomycin administration setting } \\
\hline Inpatient ${ }^{\mathrm{b}}$ & 249 (99) & $84(97)$ & $30(94)$ & 384 (98) \\
\hline Outpatient $\mathrm{t}^{\mathrm{b}}$ & $59(24)$ & $40(46)$ & $9(28)$ & $109(28)$ \\
\hline $\begin{array}{l}\text { Intensive care unit anytime } \\
\text { during daptomycin treatment }{ }^{\mathrm{b}}\end{array}$ & $93(37)$ & $19(22)$ & $10(31)$ & $129(33)$ \\
\hline \multicolumn{5}{|l|}{ Underlying diseases $^{c}$} \\
\hline Hypertension & $144(57)$ & $59(68)$ & $15(47)$ & $233(59)$ \\
\hline Diabetes mellitus & $116(46)$ & $49(56)$ & $19(59)$ & $196(50)$ \\
\hline Heart failure & $54(22)$ & $12(14)$ & $3(9)$ & 75 (19) \\
\hline Anemia, all hematologic disease & $40(16)$ & $16(18)$ & $3(9)$ & $64(16)$ \\
\hline Peripheral vascular disease & $26(10)$ & $17(20)$ & $6(19)$ & $50(13)$ \\
\hline Sepsis & $36(14)$ & $7(8)$ & $2(6)$ & $47(12)$ \\
\hline Cancer $^{\mathrm{b}}$ & $26(10)$ & $9(10)$ & $8(25)$ & $45(11)$ \\
\hline Acute coronary syndrome & $28(11)$ & $8(9)$ & $3(9)$ & $40(10)$ \\
\hline
\end{tabular}

${ }^{a}$ Includes 23 patients with unreported dosing frequency or who received a single dose of daptomycin.

${ }^{\mathrm{b}} \mathrm{p}<0.05$ for every 48 hrs vs 3 times/wk vs every 24 hrs ( $\chi^{2}$ test).

'Each patient could have had more than one underlying disease; only underlying diseases that were $\geq 10 \%$ overall are shown.

and verified by the investigators at each site. For the purpose of this analysis, the safety of patients receiving intermittent hemodialysis was assessed overall and based on their final dosing frequency of daptomycin administration when available: every 48 hours, 3 times/week, or every 24 hours.

\section{Safety Assessment}

All patients who received any dose or dosing frequency of daptomycin were included in the safety analysis. Throughout the daptomycin treatment period and up to 30 days after the last dose of daptomycin, changes in physical findings, clinical signs and symptoms, and laboratory values consistent with serious and nonserious adverse events were to be documented. Adverse events were considered serious if they resulted in any of the following: death, a life-threatening state, disability or incapacity, hospitalization, congenital anomaly or birth defect, or an important medical event.

The following additional information was collected for all adverse events: day of onset relative to daptomycin initiation, severity (mild, moderate, severe), relationship to daptomycin (not related, possibly related), action taken with daptomycin (none, stopped permanently, dose reduced, stopped temporarily), other action taken (none, concomitant drug, procedure performed, other), outcome (resolved, resolved with residual effects, death, ongoing, unknown), and resolution (day of resolution or day of last contact relative to daptomycin start). All adverse events were determined by the investigators by using International Committee on Harmonisation E2A definitions of seriousness and severity. ${ }^{22}$

\section{Statistical Analysis}

Continuous variables were reported as mean \pm $\mathrm{SD}$ or median and range. Categoric variables 
Table 2. Types of Primary Infection in the Study Patients According to Daptomycin Dosing Schedule

\begin{tabular}{|c|c|c|c|c|c|}
\hline \multirow[b]{3}{*}{ Infection Type } & \multicolumn{4}{|c|}{ No. (\%) of Patients } & \multirow[b]{3}{*}{$\mathrm{p}$ Value } \\
\hline & \multicolumn{3}{|c|}{ Daptomycin Dosing Schedule } & \multirow[b]{2}{*}{$\begin{array}{l}\text { All Patients }{ }^{a} \\
\qquad(\mathrm{n}=393)\end{array}$} & \\
\hline & $\begin{array}{c}\text { Every } 48 \text { Hours } \\
(n=251)\end{array}$ & $\begin{array}{c}3 \text { Times/Week } \\
(\mathrm{n}=87)\end{array}$ & $\begin{array}{c}\text { Every } 24 \text { Hours } \\
(\mathrm{n}=32)\end{array}$ & & \\
\hline \multicolumn{6}{|l|}{ Bacteremia } \\
\hline Catheter-related & 99 (39) & $39(45)$ & $5(16)$ & 154 (39) & 0.01 \\
\hline Non-catheter-related & $48(19)$ & $12(14)$ & $7(22)$ & $70(18)$ & 0.46 \\
\hline Skin and skin structure & $40(16)$ & $14(16)$ & $12(38)$ & $70(18)$ & 0.01 \\
\hline Endocarditis & $17(7)$ & $9(10)$ & $3(9)$ & $29(7)$ & 0.53 \\
\hline Bone and joint & $18(7)$ & $6(7)$ & $2(6)$ & $27(7)$ & 0.98 \\
\hline Urinary tract or pyelonephritis & $17(7)$ & $5(6)$ & $2(6)$ & $26(7)$ & 0.94 \\
\hline Other ${ }^{b}$ & $12(5)$ & $2(2)$ & $1(3)$ & $17(4)$ & 0.58 \\
\hline
\end{tabular}

${ }^{2}$ Includes 23 patients with unreported dosing frequency or who received a single dose of daptomycin.

bOther includes the following: 9 not specified infections, 3 necrotizing infections, 1 not specified foreign device infection, 1 central nervous system infection, and 1 nonsurgical antibiotic prophylaxis.

were reported as the number and percentage of patients with the reported characteristic. Continuous variables were compared by using the Student $t$ test, Mann-Whitney U test (Wilcoxon rank-sum), and one-way analysis of variance, where appropriate. Categoric comparisons were performed with the $\chi^{2}$ test or Fisher exact test. Statistical significance was defined as a p value of 0.05 or less. Statistical analysis was performed by using JMP statistical software, version 7, and SAS statistical software, version 9.1 (SAS Institute, Inc., Cary, NC). This analysis was not powered to address a particular safety outcome.

\section{Results}

\section{Patient Demographics}

Of a total of 4554 patients available in the registry from 2005-2008, 393 (9\%) received intermittent hemodialysis and were included in this analysis. These patients were treated at 54 different CORE sites, mostly (46\%) large teaching hospitals. Overall, 251 patients (64\%) received a final daptomycin frequency of every 48 hours. The remainder received treatment either 3 times/ week (87 patients [22\%]) or every 24 hours (32 patients [8\%]), or dosing frequency was not reported or patients received only a single dose of daptomycin (23 patients [6\%]). Initial and final daptomycin dosing frequencies were unchanged in 365 patients (93\%). Demographic data for all patients and stratified by daptomycin final dosing schedule are detailed in Table 1.

In the hemodialysis population overall, more than half of the patients were female, older than 51 years, and in the hospital 2 days before daptomycin administration (Table 1). Almost all patients received daptomycin as an inpatient, and $33 \%$ of all patients received some part of their daptomycin treatment in an intensive care unit setting. Hypertension and diabetes mellitus were the most common underlying diseases. No significant differences were seen in the demographics among groups by daptomycin dosing frequency except that the every-48-hours group had the highest rate of daptomycin treatment in an intensive care unit and the every-24-hours group had the highest predominance of cancer.

\section{Infection Types and Pathogens}

Most patients were treated for bacteremia; skin and skin structure infection was the next most common type of infection. No significant differences were noted among daptomycin dosing frequency groups when categorized by primary infection types except for catheterrelated bacteremia and skin and skin structure infections (Table 2). The primary causative pathogen was S. aureus (155 patients [39\%]), with 117 patients (30\%) having MRSA. Although the methodology varied by institution, the daptomycin MICs for S. aureus, reported for 20 patients, ranged from $0.09-1 \mathrm{mg} / \mathrm{L}$. The vancomycin MICs for S. aureus, reported for 105 patients, ranged from less than or equal to 0.2 $\mathrm{mg} / \mathrm{L}$ to $3 \mathrm{mg} / \mathrm{L}$ (19 patients [18\%] had MICs > 1 $\mathrm{mg} / \mathrm{L})$. Enterococci were cultured in 143 patients (36\%); vancomycin-resistant enterococci were present in 108 patients (27\%), most of which were Enterococcus faecium (77 patients [20\%]). 
Table 3. Adverse-Event Rates in the Study Patients by Daptomycin Dosing Schedule

\begin{tabular}{|c|c|c|c|c|c|}
\hline \multirow[b]{3}{*}{ Characteristic } & \multicolumn{4}{|c|}{ No. (\%) of Patients } & \multirow[b]{3}{*}{ p Value ${ }^{b}$} \\
\hline & \multicolumn{3}{|c|}{ Daptomycin Dosing Schedule } & \multirow[b]{2}{*}{$\begin{array}{l}\text { All Patients }{ }^{a} \\
\quad(n=393)\end{array}$} & \\
\hline & $\begin{array}{l}\text { Every } 48 \text { Hours } \\
(\mathrm{n}=251)\end{array}$ & $\begin{array}{c}3 \text { Times/Week } \\
(\mathrm{n}=87)\end{array}$ & $\begin{array}{l}\text { Every } 24 \text { Hours } \\
(\mathrm{n}=32)\end{array}$ & & \\
\hline Any adverse event & $63(25)$ & $14(16)$ & $10(31)$ & $94(24)$ & 0.13 \\
\hline Any possibly related adverse event & $17(7)$ & $7(8)$ & $2(6)$ & $28(7)$ & 0.91 \\
\hline Any serious adverse event & $38(15)$ & $9(10)$ & $4(13)$ & $55(14)$ & 0.52 \\
\hline $\begin{array}{l}\text { Any serious and possibly related adverse } \\
\text { event }\end{array}$ & $3(1)$ & $2(2)$ & $0(0)$ & $6(2)$ & 0.59 \\
\hline Discontinuation due to adverse event & $12(5)$ & $3(3)$ & $2(6)$ & $20(5)$ & 0.79 \\
\hline Deaths & $31(12)$ & $5(6)$ & $3(9)$ & $42(11)$ & 0.22 \\
\hline \multicolumn{6}{|l|}{ Most common adverse events by } \\
\hline \multicolumn{6}{|l|}{ MedDRA preferred term $(>1 \% \text { overall })^{c}$} \\
\hline Hypotension & $7(2.8)$ & $2(2.3)$ & $1(3.1)$ & $10(2.5)$ & \\
\hline Increased blood CK level & $4(1.6)$ & $2(2.3)$ & $2(6.3)$ & $8(2.0)$ & \\
\hline Cardiorespiratory arrest & $5(2.0)$ & $1(1.1)$ & $0(0)$ & $7(1.8)$ & \\
\hline Diarrhea & $7(2.8)$ & $0(0)$ & $0(0)$ & $7(1.8)$ & \\
\hline Rash & $4(1.6)$ & $0(0)$ & $0(0)$ & $5(1.3)$ & \\
\hline Respiratory failure & $5(2.0)$ & $0(0)$ & $0(0)$ & $5(1.3)$ & \\
\hline Sepsis & $3(1.2)$ & $0(0)$ & $1(3.1)$ & $5(1.3)$ & \\
\hline Cardiac arrest & $3(1.2)$ & $0(0)$ & $1(3.1)$ & $4(1.0)$ & \\
\hline Septic shock & $3(1.2)$ & $0(0)$ & $0(0)$ & $4(1.0)$ & \\
\hline Thrombocytopenia & $3(1.2)$ & $1(1.1)$ & $0(0)$ & $4(1.0)$ & \\
\hline \multicolumn{6}{|l|}{$\begin{array}{l}\text { Most common possibly related } \\
\text { adverse events }(>0.5 \% \text { overall })^{c}\end{array}$} \\
\hline Increased blood CK level & $3(1.2)$ & $2(2.3)$ & $2(6.3)$ & $7(1.8)$ & \\
\hline Rash & $3(1.2)$ & $0(0)$ & $0(0)$ & $4(1.0)$ & \\
\hline Eosinophilia & $2(0.8)$ & $1(1.1)$ & $0(0)$ & $3(0.8)$ & \\
\hline Diarrhea & $2(0.8)$ & $0(0)$ & $0(0)$ & $2(0.5)$ & \\
\hline Hyponatremia & $1(0.4)$ & $1(1.1)$ & $0(0)$ & $2(0.5)$ & \\
\hline Pyrexia & $1(0.4)$ & $1(1.1)$ & $0(0)$ & $2(0.5)$ & \\
\hline Thrombocytopenia & $2(0.8)$ & $0(0)$ & $0(0)$ & $2(0.5)$ & \\
\hline
\end{tabular}

MedDRA = Medical Dictionary for Regulatory Activities; $\mathrm{CK}=$ creatine kinase

ancludes 23 patients with unreported dosing frequency or who received a single dose of daptomycin.

${ }^{b}$ Calculated only for patients with known dosing frequency (every 48 hrs vs 3 times/wk vs every 24 hrs).

'Each patient could have had more than one adverse event.

The daptomycin MICs for Enterococcus species, reported in 22 patients, ranged from $0.19-4$ $\mathrm{mg} / \mathrm{L}$.

\section{Previous and Concomitant Antibiotic Therapy}

Three hundred nine patients (79\%) had been treated previously with an average of 1.3 antibiotics. In these patients, vancomycin was used most frequently (213 patients [69\%]), followed by $\beta$-lactams (77 [25\%]), linezolid (61 [20\%]), aminoglycosides (40 [13\%]), fluoroquinolones (41 [13\%]), and carbapenems (18 [6\%]); other antibiotics were used in 41 patients (13\%). There were no significant differences in use of previous antibiotics among daptomycin dosing frequency groups. The most common reason for discontinuation of a previous antibiotic was failure (112 patients [36\%]). Failure was documented as a contributing reason to discontinuation in 72 (34\%) of the 213 patients receiving previous vancomycin. The most common reasons for discontinuing $\beta$-lactams were failure (20/77 patients [26\%]) and resistant pathogens (19/77 [25\%]). Linezolid discontinuation reasons were primarily failure (19/61 [31\%]) and toxicity or intolerance (16/61 [26\%]).

Two hundred forty-one patients (61\%) received daptomycin in combination with an average of 1.7 different antibiotics. Concomitant antibiotic use was not significantly different among dapto-mycin dosing frequency groups. A $\beta$-lactam was used most frequently (94 patients [39\%]), followed by an aminoglycoside (53 [22\%]), vancomycin (50 [21\%]), carbapenem (44 [18\%]), fluoroquinolone (43 [18\%]), rifampin (31 [13\%]), and linezolid (12 [5\%]); other antibiotics were used in 65 (27\%) of the patients taking concomitant antibiotics. 
Dose and Frequency of Daptomycin

The median final daptomycin dose was 6 $\mathrm{mg} / \mathrm{kg}$ (range 2-14.75 mg/kg); patients with skin and skin structure infection and/or urinary tract infections or pyelonephritis received a median of $4 \mathrm{mg} / \mathrm{kg}$ (range $2-10 \mathrm{mg} / \mathrm{kg}$ ), and those with all other infection types received a median of 6 $\mathrm{mg} / \mathrm{kg}$ (range $2-14.75 \mathrm{mg} / \mathrm{kg}$ ). The median dose in patients receiving treatment every 24 hours was $4 \mathrm{mg} / / \mathrm{kg}$ (range 2-7 mg/kg) compared with $6 \mathrm{mg} / \mathrm{kg}$ (range 3-14.75 mg/kg) in the every-48hours group, and $6 \mathrm{mg} / \mathrm{kg}$ (range $3-9.5 \mathrm{mg} / \mathrm{kg}$ ) in the 3 times/week group. By infection type, the every-24-hours group had the lowest median dose for all categories except for skin and skin structure infections and the group of other infections. Data were not collected to determine if the daptomycin dose was based on ideal or total body weight.

The median duration of therapy was 12 days (range 1-84 days). The median daptomycin duration of therapy was longest for bone and joint infections (17.5 days) and endocarditis (15 days) and was shortest for skin and skin structure infections and urinary tract infections or pyelonephritis (10 days) and the group of other infections ( 6 days). The median duration was shortest in the every-24-hours group (10.5 days) compared with the every-48-hours group (12 days) and 3 times/week group (15 days, $\mathrm{p}=0.01)$. For patients who received previous and/or follow-up antibiotics, the daptomycin duration represents a portion of the total duration of therapy.

\section{Safety}

A total of 200 adverse events occurred in 94 patients (24\%) overall, with 38 adverse events in 28 patients (7\%) classified as possibly related to daptomycin. The occurrence of adverse events including those possibly related to daptomycin was not affected significantly by dosing frequency (Table 3). Of those possibly related to daptomycin, increased blood creatine kinase level was the most common, occurring in 7 patients $(1.8 \%)$; the frequency of increased creatine kinase level was not significantly different among the dosing frequency groups. Rash and eosinophilia were reported in $4(1 \%)$ and 3 $(0.8 \%)$ patients, respectively. Diarrhea, hyponatremia, pyrexia, and thrombocytopenia occurred in 2 patients $(0.5 \%)$ each. Nine adverse events (bacterial sepsis, eosinophilia, urticaria, renal insufficiency, pancytopenia, catheter site discharge, Klebsiella infection, Pseudomonas infection, and rhabdomyolysis) in 6 patients (2\%) were reported as serious and possibly related to daptomycin. Twenty patients (5\%) discontinued daptomycin because of an adverse event; 12 (3\%) had an adverse event that was possibly related to daptomycin. In addition, no significant difference in number of patients experiencing any adverse event was observed when stratified by daptomycin dose (44/166 [27\%] for $<6 \mathrm{mg} / \mathrm{kg}$ vs $48 / 217$ [22\%] for $\geq 6$ $\mathrm{mg} / \mathrm{kg}, \mathrm{p}=0.3)$. The overall mortality rate was $11 \%$ (42/393): $12 \%(31 / 251)$ for the every-48hour group, $6 \%$ (5/87) for the 3 times/week group, 9\% (3/32) for the every-24-hour group, and $13 \%(3 / 23)$ in the remaining patients.

\section{Discussion}

In patients treated with hemodialysis, the administration of many drugs is challenging, especially those drugs that are eliminated through the kidneys. For antibiotics, this is especially problematic since appropriate dosing is necessary in order to eradicate the pathogen(s), prevent development of resistance, and avoid toxicity.

Since its introduction, daptomycin has been used frequently across a variety of infection types with good clinical outcomes. ${ }^{23}$ The data from this analysis of the CORE registry from 2005-2008 showed that the rate of adverse events from daptomycin was similar across dosing frequency groups.

There has been a need for additional safety data with daptomycin in this population as slower elimination and higher troughs might lead to higher adverse-event rates. However, rates of adverse events generally were similar to those found in randomized, multicenter trials of daptomycin. ${ }^{16,17}$ With respect to adverse events judged to be possibly related to daptomycin, only $2 \%$ were classified as serious. An elevated creatine kinase level was observed in less than $2 \%$ of patients in the population evaluated in the safety analysis. It is noteworthy that daptomycin yielded similar results across all dosing frequency groups without the need for therapeutic drug monitoring, which is often recommended for vancomycin and teicoplanin. The every-24-hour dosing interval is not recommended in patients receiving hemodialysis since the prolonged halflife will lead to higher trough concentrations than those seen in patients with normal renal function. Data accumulated to date suggest that 
daptomycin-associated muscle toxicity is related to an inadequate amount of time between doses where the minimum serum concentration may be elevated. ${ }^{24}$ The daptomycin dose was not significantly different between those who developed an elevated creatine kinase level and those who did not. It is unknown if the lower dose used in patients receiving daptomycin every 24 hours may have mitigated the occurrence of adverse events. Further data are expected from an ongoing prospective study of patients with moderate or severe renal dysfunction comparing daptomycin with vancomycin for patients with skin and skin structure infections or S. aureus bacteremia. ${ }^{25}$

The overall mortality rate of $11 \%$ in this analysis is similar to published data in this population. One group of authors investigated the outcomes of first-time infections in hospitalized patients receiving hemodialysis. ${ }^{26}$ An outcome of death occurred in $13.8 \%$ of firsttime infections. The types of infections were similar in frequency to those described in this analysis, with a preponderance of bacteremia and access-related infections and lower rates of cardiac, skin, and urinary tract infections. One notable difference was the absence of pulmonary infections in the CORE registry due to daptomycin's lack of efficacy in this setting. ${ }^{20}$

\section{Limitations}

The retrospective nature of the registry has several important limitations that prevent drawing firm conclusions regarding the safety of daptomycin in the treatment of gram-positive infections in the hemodialysis population. There was no control group; all patients in the registry had received daptomycin in addition to other treatment options including concomitant antibiotics and surgical interventions as appropriate. Many variables were not controlled, as patients were managed at the discretion of their physicians, which, while a limitation, also provides real-world perspective. Thus, safety outcomes and mortality may have been influenced by factors other than the use of daptomycin.

The patient population was diverse, with a variety of infections and pathogens. Although reflective of real-world clinical practice, the number of subjects in any particular subgroup was small. Susceptibility data were incomplete, making it difficult to identify any association between in vitro activity of daptomycin and mortality. There was no patient follow-up after the completion of daptomycin therapy; thus, identifying late adverse events was not possible. Finally, details on the administration of hemodialysis, information on different variables such as duration, type of filter, and flow rates were not collected as part of the CORE registry during this time period. These and other factors may alter significantly the amount of drug clearance during a dialysis session. ${ }^{27}$ This information was captured in 2009, and $66 \%$ of patients undergoing intermittent dialysis received their daptomycin after dialysis. Although the product labeling of daptomycin recommends administration after hemodialysis sessions, ${ }^{20}$ strict assurance that study patients received it in such a manner was not captured.

\section{Conclusion}

The safety data in this analysis of daptomycin in patients receiving hemodialysis who had a variety of gram-positive infections suggest that daptomycin as part of the standard of care in this setting may be a well-tolerated treatment option. The adverse events reported during daptomycin treatment appeared similar across all doses and dosing frequencies that are commonly used to treat infections in patients receiving hemodialysis, including 3 times/week. Further study in prospective clinical trials is warranted.

\section{Acknowledgments}

The authors gratefully acknowledge the contributions of the CORE investigators and the institutions from which the data were collected. The authors would like to thank Lawrence Friedrich, Pharm.D., Cubist Pharmaceuticals, Inc., and Susan DePetris, Ph.D., Phase Five Communications Inc., for their input and assistance in the preparation of the manuscript for publication from the authors' original creation. The authors also acknowledge the support of Cubist Pharmaceuticals, Inc.

\section{References}

1. Himmelfarb J. Hemodialysis complications. Am J Kidney Dis 2005;45:1122-31

2. Foley RN. Infections in patients with chronic kidney disease. Infect Dis Clin North Am 2007;21:659-72.

3. Berman SJ, Johnson EW, Nakatsu C, Alkan M, Chen R, LeDuc J. Burden of infection in patients with end-stage renal disease requiring long-term dialysis. Clin Infect Dis 2004;39:1747-53

4. U.S. Centers for Disease Control and Prevention. Invasive methicillin-resistant Staphylococcus aureus infections among dialysis patients: United States, 2005. MMWR Morb Mortal Wkly Rep 2007;56:197-9.

5. Rybak MJ. The pharmacokinetic and pharmacodynamic properties of vancomycin. Clin Infect Dis 2006;42(suppl 1):S35-9. 
6. Hidayat LK, Hsu DI, Quist R, Shriner KA, Wong-Beringer A. High-dose vancomycin therapy for methicillin-resistant Staphylococcus aureus infections: efficacy and toxicity. Arch Intern Med 2006;166:2138-44.

7. MacLayton DO, Suda KJ, Coval KA, York CB, Garey KW Case-control study of the relationship between MRSA bacteremia with a vancomycin MIC of $2 \mu \mathrm{g} / \mathrm{ml}$ and risk factors, costs, and outcomes in inpatients undergoing hemodialysis. Clin Ther 2006;28:1208-16.

8. Sakoulas G, Moise-Broder PA, Schentag J, Forrest A Moellering RC Jr, Eliopoulos GM. Relationship of MIC and bactericidal activity to efficacy of vancomycin for treatment of methicillin-resistant Staphylococcus aureus bacteremia. J Clin Microbiol 2004:42:2398-402.

9. U.S. Centers for Disease Control and Prevention. Staphylococcus aureus resistant to vancomycin: United States, 2002. MMWR Morb Mortal Wkly Rep 2002;51:565-7.

10. Dawn M, Sievert DM, Rudrik JT, et al. Vancomycin-resistant Staphylococcus aureus in the United States, 2002-2006. Clin Infect Dis 2008;46:668-74.

11. Malani PN, Rana MM, Banerjee M, Bradley SF. Staphylococcus aureus bloodstream infections: the association between age and mortality and functional status. J Am Geriatr Soc 2008;56: $1485-9$

12. Blot SI, Vandewoude KH, Hoste EA, Colardyn FA. Outcome and attributable mortality in critically ill patients with bacteremia involving methicillin-susceptible and methicillinresistant Staphylococcus aureus. Arch Intern Med 2002;162: 2229-35.

13. Kang CI, Song JH, Ko KS, Chung DR, Peck KR, for the Asian Network for Surveillance of Resistant Pathogens (ANSORP) Study Group. Clinical features and outcome of Staphylococcus aureus infection in elderly versus younger adult patients. Int J Infect Dis 2011;15:e58-62.

14. Mascio CTM, Alder JD, Silverman JA. Bactericidal action of daptomycin against stationary-phase and nondividing Staphylococcus aureus cells. Antimicrob Agents Chemother 2007;51:4255-60.

15. Sader HS, Fritsche TR, Jones RN. Antimicrobial activity of daptomycin and selected comparators tested against bloodstream Staphylococcus aureus isolates from hemodialysis patients Int J Infect Dis 2009;13:291-5.

16. Arbeit RD, Maki D, Tally FP, Campanaro E, Eisenstein BI. The safety and efficacy of daptomycin for the treatment of complicated skin and skin-structure infections. Clin Infect Dis
2004;38:1673-81.

17. Fowler VG Jr, Boucher HW, Corey GR, et al. Daptomycin versus standard therapy for bacteremia and endocarditis caused by Staphylococcus aureus. N Engl J Med 2006;355:653-65.

18. Dvorchik B, Arbeit RD, Chung J, Liu S, Knebel W, Kastrissios H. Population pharmacokinetics of daptomycin. Antimicrob Agents Chemother 2004;48:2799-807.

19. Salama NH, Segal JH, Churchwell MD, et al. Intradialytic administration of daptomycin in end stage renal disease patients on hemodialysis. Clin J Am Soc Nephrol 2009;4; $1190-4$.

20. Cubist Pharmaceuticals. Cubicin (daptomycin for injection) full prescribing information. Available from http://www.cubicin. com/pdf/PrescribingInformation.pdf. Accessed December 17, 2010.

21. Rolston KVI, Segreti J, Lamp KC, Friedrich LV. Cubicin outcomes registry and experience (CORE) methodology. Am J Med 2007;120(10A):S4-5.

22. U.S. Food and Drug Administration. Guideline for industry, clinical safety data management: definitions and standards for expedited reporting. Available from http://www.fda.gov/ downloads/Drugs/GuidanceComplianceRegulatoryInformation/ Guidances/UCM073087.pdf. Accessed December 17, 2010.

23. Weis F, Beiras-Fernandez A, Schelling G. Daptomycin, a lipopeptide antibiotic in clinical practice. Curr Opin Investig Drugs 2008;9:879-84

24. Bhavnani SM, Rubino CM, Ambrose PG, Drusano GL. Daptomycin exposure and the probability of elevations in the creatine phosphokinase level: data from a randomized trial of patients with bacteremia and endocarditis. Clin Infect Dis 2010;50:1568-74.

25. Cubist Pharmaceuticals, Inc. Prospective multicenter randomized evaluator-blinded comparator-controlled study to describe the safety and efficacy of daptomycin for the treatment of CSSSI and Staphylococcus aureus bacteremia among subjects with renal impairment. ClinicalTrials.gov. Bethesda, MD: National Library of Medicine. Available from http://clinical trials.gov/show/NCT01104662. Accessed August 10, 2010.

26. Allon M, Radeva M, Bailey J, et al. The spectrum of infectionrelated morbidity in hospitalized haemodialysis patients. Nephrol Dial Transplant 2005;20:1180-6.

27. Decker BS, Mueller BA, Sowinski KM. Drug dosing considerations in alternative hemodialysis. Adv Chronic Kidney Dis 2007;14:e17-26. 